\title{
Taxonomic trees of fluidic oscillators
}

\author{
Václav Tesař ${ }^{1, a}$ \\ ${ }^{1}$ Institute of Thermomechanics v.v.i., Czech Academy of Sciences \\ Dolejškova 5, 18200 Praha-Kobylisy, Czech Republic
}

\begin{abstract}
Fluidic no-moving-part oscillators generating pulsation in fluid flow became recently a very popular subject of investigations. The reason is their capability to increase efficiency of various chemical and physico/chemical processes. Also control of flows past bodies is more effective with the pulsation. Advantages of fluidic oscillators used for the task are low cost, robustness, long life, no maintenance, and other similar factors associated with absence of mechanical components. New oscillator principles - as well as old, nearly forgotten and now re-discovered - are currently developed. Sheer number of possible alternatives makes them difficult to survey. This paper attempts at clarifying the situation.
\end{abstract}

\section{Introduction}

Dictionary definition says:

Taxonomy = classification into groups based on similarities of structure or relationships.

Fluidic oscillators are devices supplied by a steady fluid flow and converting it into a self-excited periodic flow. Although some of their working principles were known (or at least their existence was recognised) already in $19^{\text {th }}$ century [2], the configurations as we know them today were designed, tested, and patented later, around the middle of the $20^{\text {th }}$ century. The inventors, Warren [8], Zalmanzon [9], and others were then guided by knowledge then already acquired with electronic oscillators - consisting of an electronic amplifier and electrically conducting loop forming a negative feedback signal path. In fluidics, the configurations were analogous: a fluidic oscillator consists of at least one fluidic amplifier together with channels for feedback loop fluid flows. From their originally expected applications, control and signal processing systems, fluidics was displaced by electronics and, became practically forgotten. A new lease of life they obtained recently when they were found useful for generating pulsation in various chemical and physico/chemical process engineering operations, the efficiency of which was demonstrated to be significantly improved by the pulsation superposed on the flow. Also in control of fluid flows past objects, e.g. by suppressing turbulence or flow separation on aircraft wings, the acting control jets are substantially more efficient if they pulsate and this may be achieved with fluidics. Compared with a mechanical counterparts the fluidic oscillators exhibit lower cost, easy manufacturing, robustness, long life, no maintenance, and other beneficial factors stemming from the absence of mechanical, moved or deformed valve components.

\footnotetext{
$\overline{\text { a Corresponding author: tesar@it.cas.cz }}$
}

The growing demand has led to inventing various new oscillator working principles - as well as studying agsin the old ones. Number of existing and possible alternatives has now reached a stage making orientation in this field difficult. Considering possible combinations and permutations makes them and the relations between them quite confusing. The present survey paper is an attempt to clarify the situation.

\section{Standard: amplifier and loop(s)}

Most present-day fluidic oscillators consist from two separately identifiable components: a no-moving-part fluidic amplifier - mostly of the bistable diverting type, as shown in Fig. 1 - and one or two feedback loop channels for fluid flow. The bistable two-loop configuration (Fig.1) may be seen as two monostable devices of the block diagram in Fig. 2, with common supply nozzle.

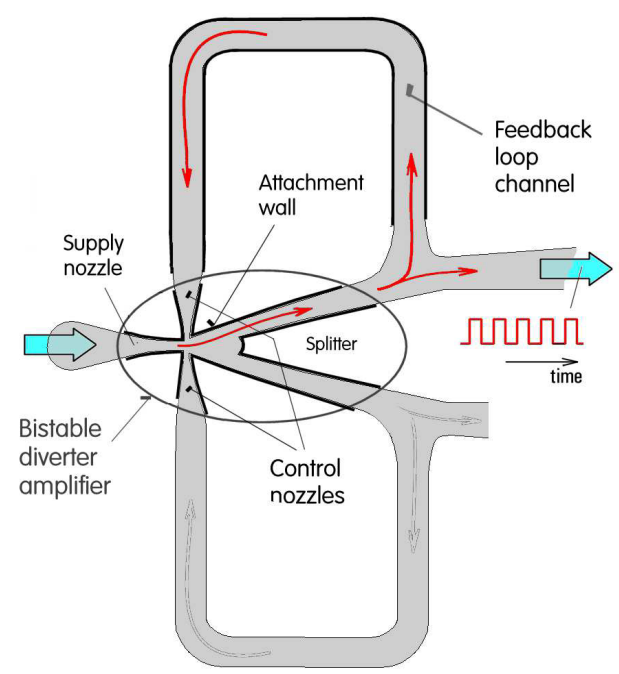

Figure 1 Oscillators with bistable jet-deflection amplifier and two feedback loops as shown here are now practically standard. 


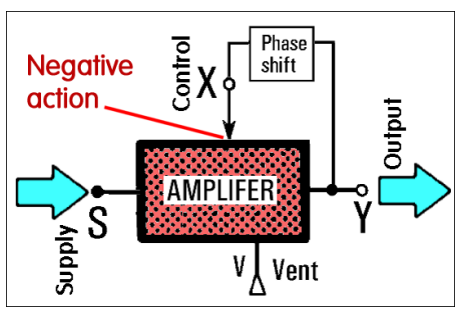

Figure 2 Basic principle of self-excited oscillation with amplifier and negative feedback loop. The design in Fig. 1 may be viewed upon as an anti-parallel configuration with common supply nozzle of two integrated amplifiers \& feedbacks.

The bistability is capability of the jet (generated in supply nozzle) to stay deflected in one of two different regimes without any external deflecting action. In the jettype amplifier it is the consequence of the Coanda attachment effect: the jet clings to a nearby suitably placed solid wall. The negative feedback flow tends to separate the jet from its wall, so that it cannot remain steady and oscillates between the two regimes. Its use in the oscillator means the generated output flow has the character of a train of more or less rectangular pulses (Fig. 1). It is possible to use instead the proportional, continuously operating amplifier - generating a harmonic outp[ut flow - but the versions with jet attachment are generally more reliable and easier to set up.

There are two alternative possibilities, shown in Fig. 2, how to achieve the jet separation from the wall to which it is attached. For the explanation the picture uses a simpler amplifier version, with asymmetric geometry and only one attachment wall (so that it is monostable). In addition to the mono- and bistable versions, Fig. 3 shows yet another factor that multiplies the number of potential amplifier alternatives. There are two cases, $A$ and $B$. The first $A$ is presented in the top part of the illustration. At it is shown with the jet attached while at right the jet is displaced by the flow through the control nozzle oriented perpendicularly. This control action is more common. In the bottom part of Fig. 3 is presented the other alternative B. The separation of the jet from the wall is there achieved by making it more difficult for the main flow to get into the exit terminal. This is similar to the effect

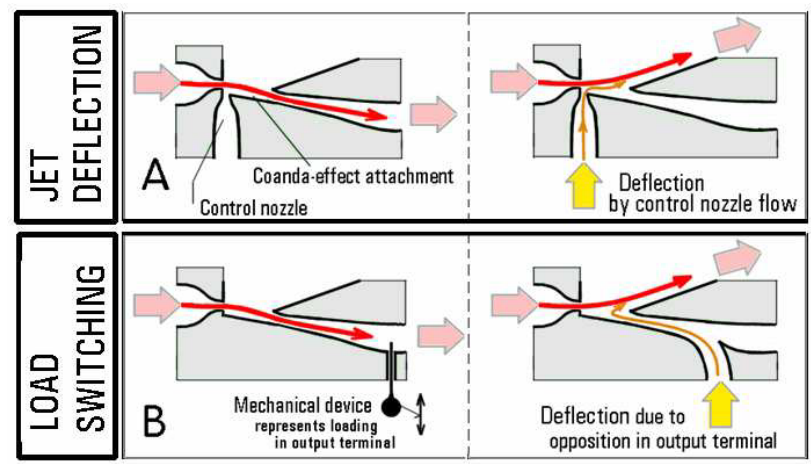

Figure 3 Explained here on the example of simple one-sided monostable amplifier are the two alternative modes, A and B, of controlling the Coanda attachment. Apart from the more usual A, jet separation from the wall by a perpendicular control nozzle flow, the jet may be also forced away by blocking, B, the flow in output terminal.

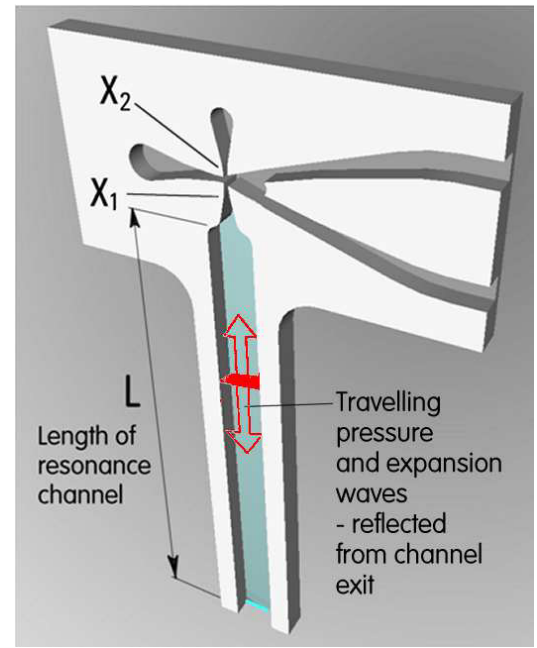

Figure 4 Recently introduced [1] oscillator principle using travelling waves in a resonator. It is an attractive solution for high oscillation frequencies

achieved by insertion of a mechanical flow-blocking device as is schematically represented in the left-hand drawing $B$ in Fig. 3.

Adding to the number of possible oscillator alternatives is another type of feedback presented in Fig. 4. There is no channel forming a loop. The negative feedback action is generated in a resonator channel (or tube - or any resonant cavity) attached to thet control nozzle $\mathrm{X}_{1}$. Instead of running a flow around the loop the feedback action here results from the return of compression and expansion waves reaching the ends of the resonator. The pressure wave at the end of the resonator generates an rarefaction wave moving by the

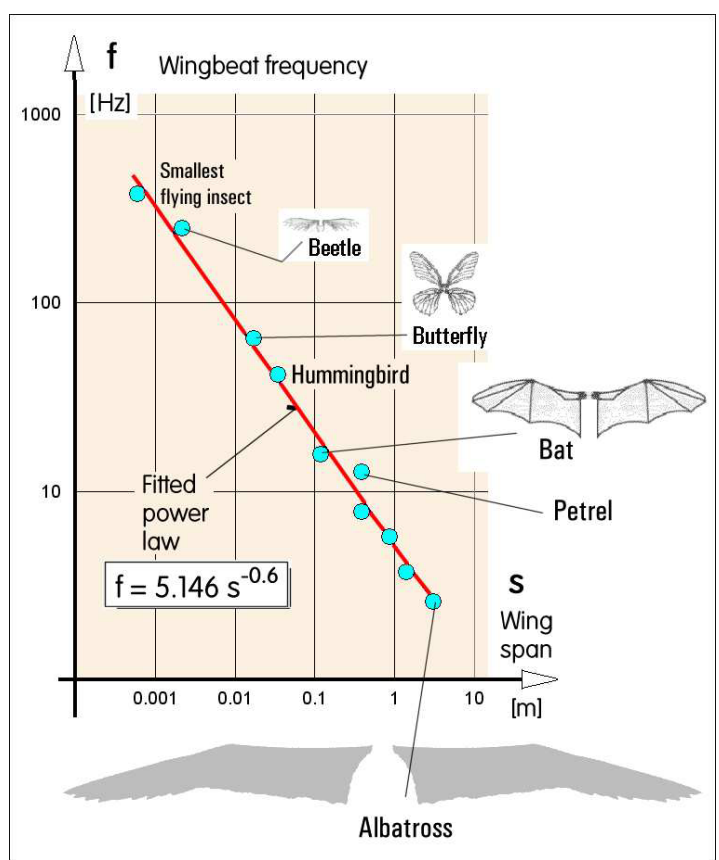

Figure 5 Universal law of nature: higher frequency oscillation is generated in smaller sized oscillator. It is valid not only for flying organisms as shown here, but applies also to fluidic oscillators. 


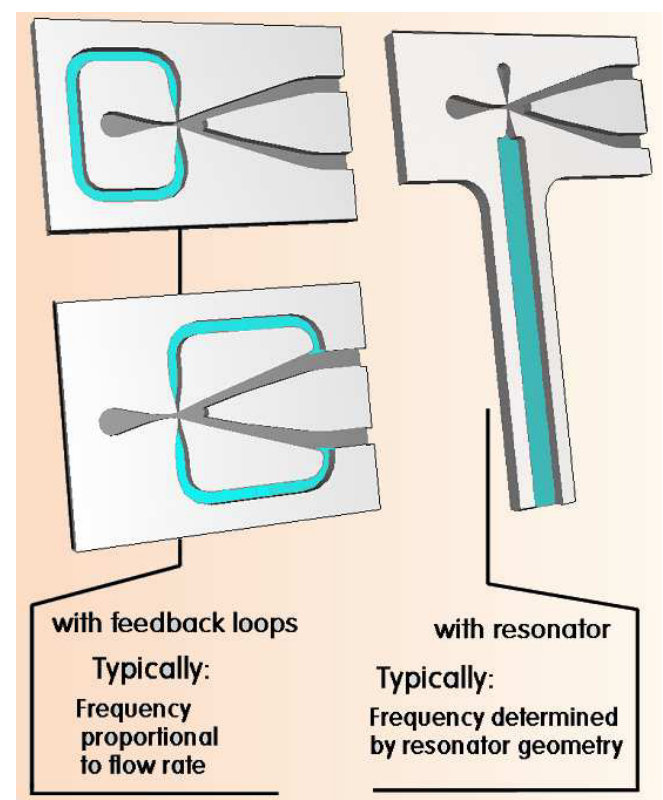

Figure 6 Two basic behaviour modes of fluidic oscillators with bistable diverter amplifiers: either constant Strouhal number [2] (at left) or constant frequency (right).

speed of back to the amplifier. There its arrival causes jet separation from its attachment wall. Of course, this travelling wave character of the feedback, may be applied not only with the bistable amplifier but also in other configurations (such as the monostable amplifier or load-switching from Fig. ). As a result, the number of possible combinations increases.

The reason for use of the acoustic feedback is the capability to achieve high oscillation frequency, which is desirable in some applications. Sound waves propagate rather fast and the resonating channel may be quite short, both factors contributing to the high frequency. In the feedback loop versions typical top frequency in a palmtop size oscillator [1] is somewhere near to $f=300-$ $400 \mathrm{~Hz}$. Higher frequency would require high flow velocities and very small size, both inconvenient factors for some oscillator uses. The problem with the small characteristic size scale $\mathrm{s}$ is explained by the interesting general law presented in Fig. 6,

$$
\mathrm{f} \sim \mathrm{s}^{-0.6}
$$

valid in nature universally for oscillating objects - and in principle also valid for the fluidic oscillators with feedback loops governed by the Strouhal number constancy (Fig. 6).

There is yet another factor complicating any survey of oscillators. It is the alternative configuration with only a single feedback loop channel connecting both control nozzles (top left in Fig. 6). This not immediately apparent configuration used to be attributed to Spyropoulos [9] but it is in fact another invention of Warren [10]. While requiring less space and simpler control circuit it is less reliable and more difficult to get operating. Increasing the number of possible permutations in general surveys of oscillators, at adds to the complexity of the taxonomic tree discussed here.

\section{Less known feedbacks}

Apart from the most popular designs presented in Fig. 6, there are many other self-excited fluid flow oscillation phenomena that may be also used in fluidics. The reason for their slower acceptance was absence of obvious feedback mechanisms and general difficulties associated with their identification - compared with the immediately apparent feedback loop principle analogy to the electronic oscillators.

A similar direct analogy exists also between the negative-slope oscillator in Fig. 7 and the electronic Esaki diode oscillator [16]. The fluidic version is rarely used and in fact little known.

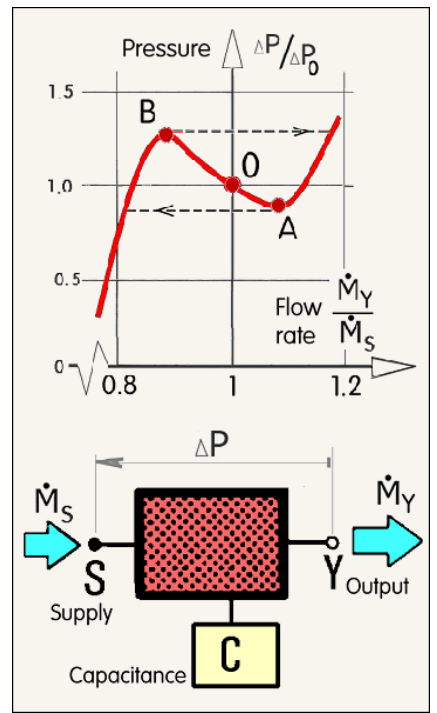

Figure 7 Hydrodynamic instability with consequent selfexcited oscillation exists in fluidic devices exhibiting negative slope on a part of their characteristic.

Similar characteristics with negative slope sections but having no electronic counterpart - are encountered in the colliding jets, Fig. 8. The tendency towards oscillation is there so strong that investigating experimentally the part of the curve between $A$ and $B$ is rather difficult and calls for efficient flow regulators. This oscillator is not used in the typical oscillator role,

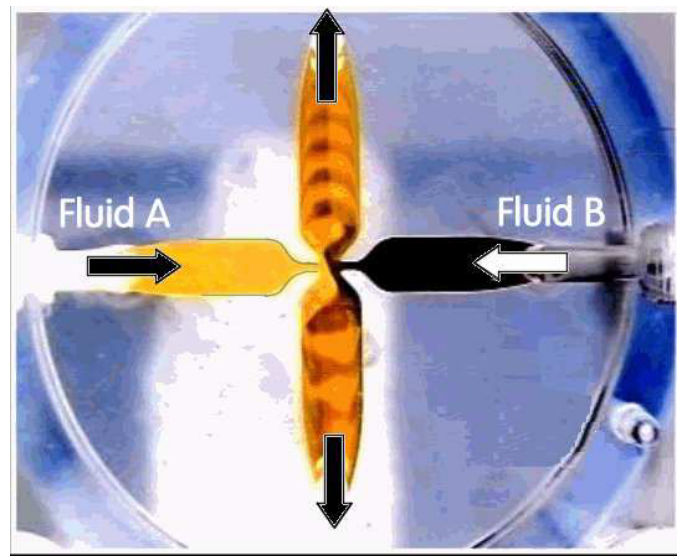

Figure 8 Hydrodynamic instability (and oscillation) is encountered also in the flowfield of colliding jets [6]. 
details of the processes taking place in the interaction space were instead investigated with the intention of using the device in small-scale, microfluidic mixers [6]. Visualisations of the flowfield (with interesting procedure of posterisation of images - drastic reduction of the colour scale) have actually led to identification of the fact that the jets turn back, following what is in effect a feedback loop path.

A typical example of naturally occurring fluidic oscillator, visualisation of the flow in which is presented in Fig. 9, is the generation of periodically vortices shed downstream from a blunt body (in Fig. 9 the body is a circular cylinder). As long ago as in the $19^{\text {th }}$ century (ref. [2]) the periodicity in this wake flow was recognised and measured. However, understanding where exactly the feedback action (which obviously must be present) takes place is still rather difficult.

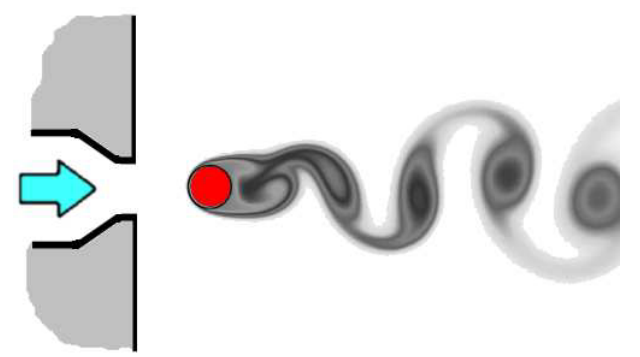

Figure 9 An example of a fluidic oscillator existing in nature - a blunt body (cylinder) with trailing von Kármán vortex street [2]. The internal feedback effect is not simply identified.

The inflow as well as outflow from the device pictured in Fig. 10 is constant - the unsteady periodic character exists only in the vicinity of the target body. This device was developed not for generating a fluidic output signal, but for use in a digital flowmeter with electronic output - electric signal generated by a periodically cooled thermistor placed at the target. The condition for success of this idea is achieving in the cavities in Fig. 10 an almost perfect constancy of Strouhal number

$$
\mathrm{Sh}=\frac{\mathrm{fb}}{\mathrm{w}}
$$

- where $b$ and $w$ are the nozzle exit width and velocity.

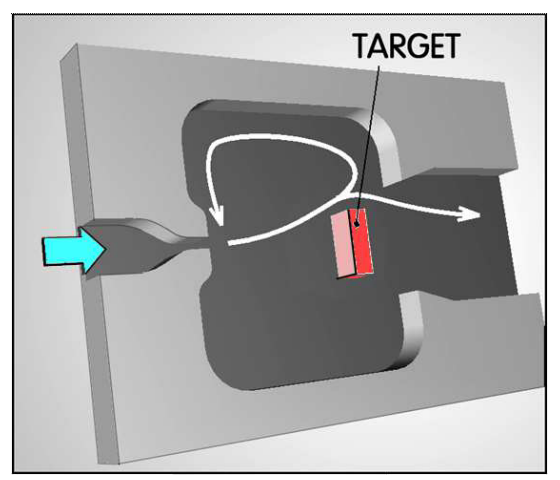

Figure 10 Oscillator with the blunt-body target, essentially similar to Fig. 9 but with oscillation enhanced by vortical motions in the cavities provided for the purpose on both sides of the jet. Device geometry was developed for $\mathrm{Sh}=$ const.
The flow rate $\dot{M}$, simply proportional to $w$, is evaluated by counting the oscillation pulses per unit of time.

Deviations from desirable perfect Strouhal number constancy at very low velocities in the flow shown in Fig. 9 were identified to stem from the Reynolds-number

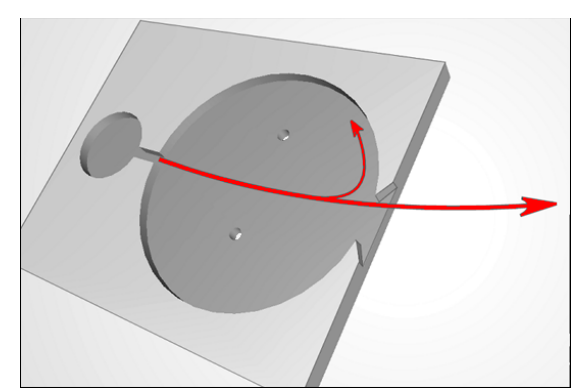

Figure 11 Nakayama oscillator [5] with internal loops driven by jet-edge separating scraper .

dependent variations of the location at which the cylinder boundary layer separates from the solid surface. This is the reason for replacing in Fig. 10 the cylinder by the target with sharp edges that fix the separation location. Stabilisation of Sh value in the flowmeters is also helped by providing the cavities in which form of alternating vortices.

At low Reynolds numbers, typical for fluidics, the vortices forming on both sides of a submerged jet can play a significant role in the oscillator behaviour. In the Nakayama oscillator [5], Fig. 11, they are peeled off by the edges of the scraper and form a vortical pattern inside the cavity, causing alternating bending of the jet.
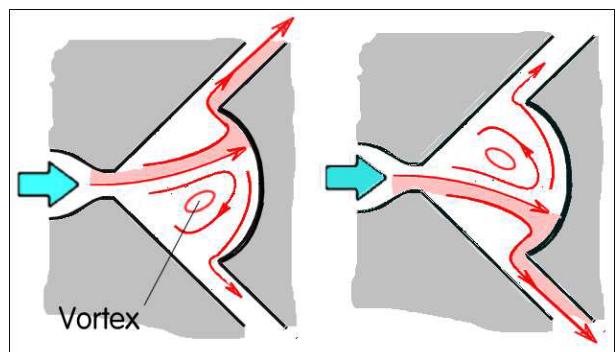

Figure 12 Naturally arising self-excited oscillatory phenomena are also based on instability of jet impingement, especially on a concave surface [3].

Another phenomena with instability and resultant periodic motions are found in impinging jets. Impingement on a flat wall is characterised by only small sideways "dancing" of the stagnation point. More powerful are the pulsations of impingement in a cavity or the concave wall as shown in Fig. 12. Pressure differences between both sides of the jet are increased if there is a suitable cavity for accommodating there the vortical motions. The vortices deflect the jet and the amplitudes of the "dance" may become quite large. Obvious disadvantage of the configuration from Fig. 12 in practical applications is poor energetic efficiency, unavoidable in flows with long submerged jet paths and sharp changes of flow directions 


\section{Relaxation oscillators}

There is a family of "feedback-less" oscillator principles little known nowadays - although one of the family members was actually the first ever patented [9] nomoving-part fluidic oscillator. The basic idea is gradually increasing level of some parameter (pressure, flow rate, or hydraulic resistance) until it reaches a limit. At thta moment the flowfield can no more keep the initial regime conditions and the flow regime collapses. The character of the flow in the collapse is such that it makes possible a return of the whole fluidic system to the starting condition. Thereafter the process of parameters increase can start again. Typically, such a collapse of the flowfield takes place in the load-switched diverter (case $B$ in Fig. 3) if its load in the active flowpath reaches the level at which the Coanda effect no more suffices in keeping the jet at its attachment wall and the jet is switched away.

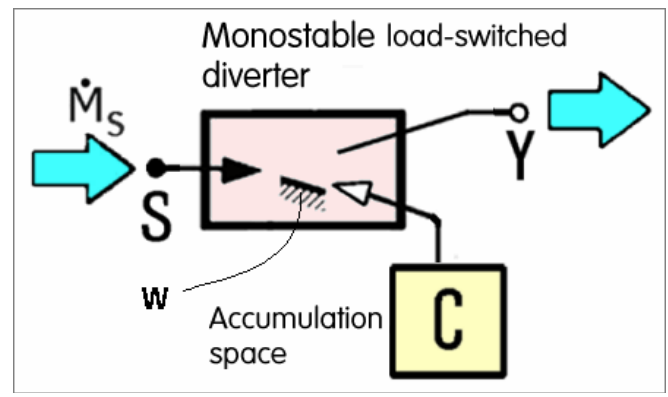

Figure 13 Schematic representation of the earliest ever patented fluidic oscillator [9] based on relaxation phenomena in monostable jet attachment.

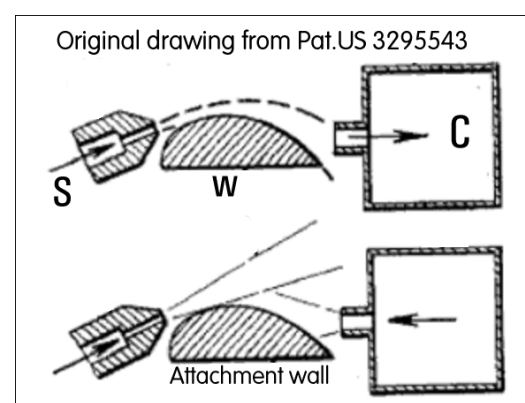

Figure 14 The tested configuration of the relaxation oscillator [9] according to Fig. 13.

Still the best example is the Zalmanzon's oscillator [9], the schematic circuit diagram of which is presented in Fig. 13. It corresponds to the drawing in Fig. 14 taken from the Patent [9], showing its origin in stalling an aeroplane wing. The monostable diverter has its single attachment wall $\mathrm{w}$ positioned so that it guides the attached jet (generated in the supply nozzle $S$ ) into the accumulation chamber $\mathrm{C}$. The fluid is air (or gas). Being compressible, it is gradually accumulated in the chamber and its pressure there increases. Of course, the accumulation is limited. Finally, the pressure in chamber $C$ reaches the level at which it represents untenable loading for the amplifier output terminal. The jet switches away from $\mathrm{W}$ and is directed into the other terminal $\mathrm{Y}-$ where it remains deflected by the return flow issuing from the chamber C. Only when the pressure in the emptied chamber reaches the starting condition, the jet returns to $\mathrm{W}$ and the chamber filling starts anew.

As a matter of fact, even in the principles described as feedback-less, analysis of the processes makes possible discovering some action that plays the feedback role.

\section{Multivibrator with two amplifiers}

\subsection{Mutual blocking of anti-parallel amplifiers}

Another little known fluidic oscillator configuration is represented schematically in Fig. 15. It corresponds to an analogous circuit in electronics called "multivibrator", [7] invented at the time of WW I. From there the idea was taken over into fluidics. It may be possible to see the bistable fluidic amplifier presented in Fig. 1, as an integral configuration of two monostable amplifiers (with common supply nozzle and also common interaction cavity, sharing also the outlet terminals).

In Fig. 15 there are the two amplifiers, in the direct analogy with electronics both of the turn-down type. They are connected in anti-parallel, so that the flow through one of them corresponds to no-flow regime in the other, opposite one. There are two main flowpaths, one from the supply terminal $S$ through the hydraulic resistor $\mathrm{R}_{1}$ and the Amplifier 1 . The other main flowpath passes through the hydraulic resistor $R_{2}$ and the Amplifier 2. If an amplifier, say the Amplifier 1, is in the OPEN regime, almost all pressure supplied from $S$ drops on the resistor $R_{1}$. The node $u_{1}$ is almost at the low, atmospheric pressure. If, however, the Amplifier 1 is in the CLOSED regime, the flow rate through $R_{1}$ is small so that the pressure drop on it is small. Almost all pressure supplied from $S$ drops on the Amplifier 1 and the node $u_{1}$ is almost at the supply pressure level. The high pressure from the node $u_{1}$ is led into the second control terminal $\mathrm{X}_{2}$ of the Amplifier 2. It tends to switch it from the OPEN regime into the CLOSED one, but this effect is delayed by the phase-shift element. Behaviour of the Amplifier 2 is

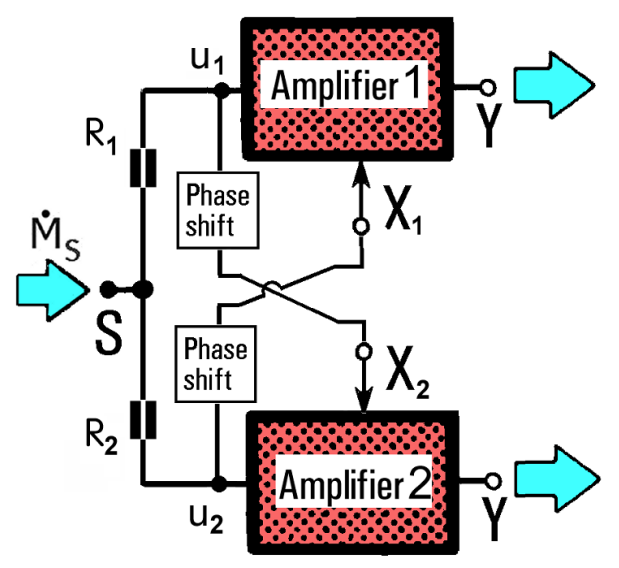

Figure 15 Fluidic oscillators may be assembled from two amplifier devices blocking each other's flow from the supply $S$ to the exit $Y$. This principle is a direct parallel to the electronic flip-flop multivibrator invented by Abraham and Bloch [7]. 
thus the very opposite to what happens in Amplifier 1, only that it is delayed. The amplifiers simply block one another. Depending on the adjustment of the phase shift, the circuit either behaves as a flip-flop binary memory or oscillates, with the pressure and flow in the output terminals $Y$ pulsating between the two regimes.

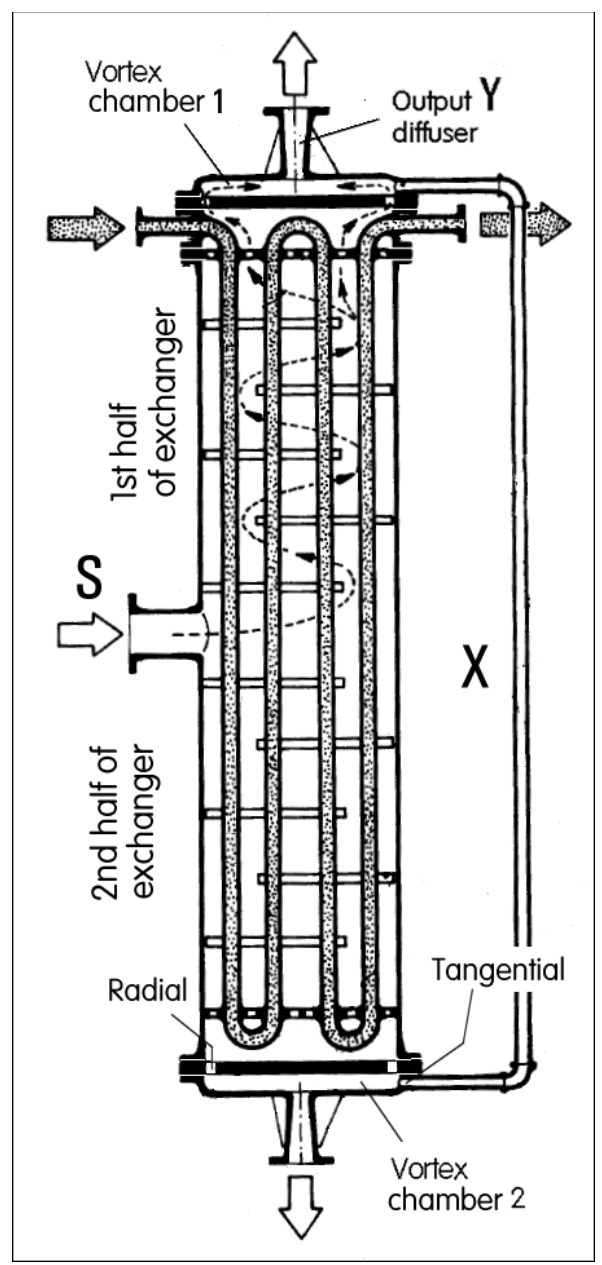

Figure 16 The heat exchanger with the flow oscillation on gas side as schematically represented in Fig. 17.

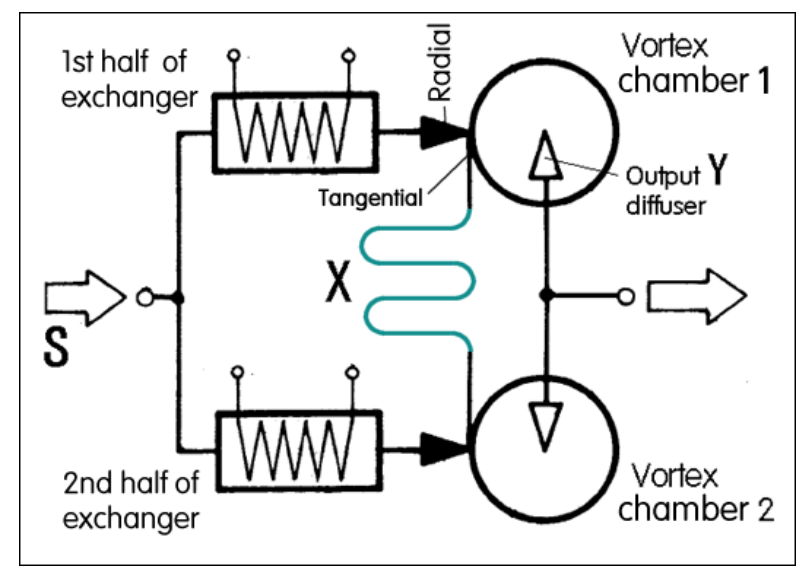

Figure 17 Heat transfer intensification by oscillation generated in a mutually blocked vortex amplifiers - actually end-spaces of exchanger body [12]. This is the earliest known fluidic oscillator based on the multivibrator principle Fig. 15.
The earliest known realisation of this oscillator principle with anti-parallel amplifiers was described in [12]. The object was a pulsating heat exchanger - the pulsation on the gas side destroying the thermal boundary layer and therefore increasing the exchanger efficiency. Upper and lower halves of the exchanger took over the role of the resistors $R_{1}$ and $R_{2}$ in Fig. 15. Spaces at ends of the echanger's cylindrically shaped body served as vortex chambers of the active devices - the vortex amplifiers.

Recently experimentally investigated oscillator presented in Fig. 18 is essentially analogous. Again, it was the turn-down capability in the amplifiers that was obtained by applying the idea of vortex amplifier. the amplifiers discussed in [15] are the CSVA version (Coanda-switched vortex amplifier) - in fact an integral combination of a bistable diverter and vortex amplifier. Upstream from the vortex chamber there are antechambers with a pair of mutually inclined attachment walls to which attaches the jet, formed by issuing from the supply nozzle.

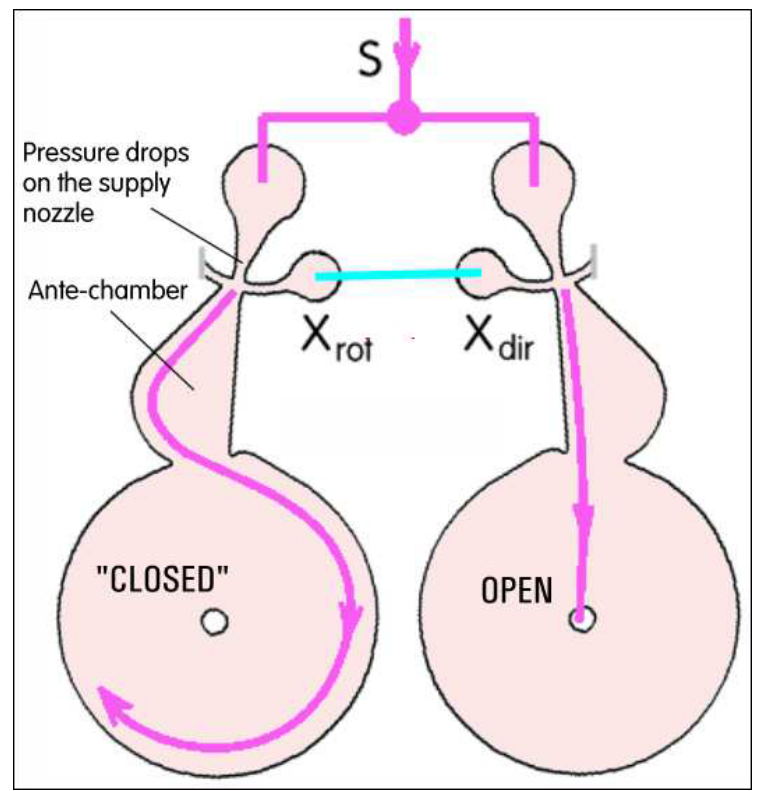

Figure 18 One version of the oscillator described in [15], with Coanda-switched turn-down valves.

Also worth noting in Fig. 18 is the fact that the large area contractions in the supply nozzles generate the pressure drops corresponding to those in Fig. 15 that were obtained by the resistors $R_{1}$ and $R_{2}$.

\subsection{Combining the ideas of relaxation and two anti- parallel devices:}

The latest designs of this type of oscillators have as their starting point the version from Fig. 18, but were developed towards simplicity. The idea is presented schematically in Fig. 19. Note that the bottom drawing there, with the monostable diverter, corresponds directly to the Zalmanzon' s design presented in Fig. 13 - only replacing the time delay by gradual compression of air in the accumulation chamber by the gradual spin-up of the 
rotation in the vortex chamber. General experience with monostable and bistable diverters prefers the former. This is why the actually built and tested was the design shown in the top part of Fig. 19. As shown in Fig. 20, the resultant fluidic oscillator was capable of operating at very low frequencies - without the inconvenient need of stowing the very extremely long feedback loop tubing, that would be necessary with the standard feedback loops of Fig. 1.

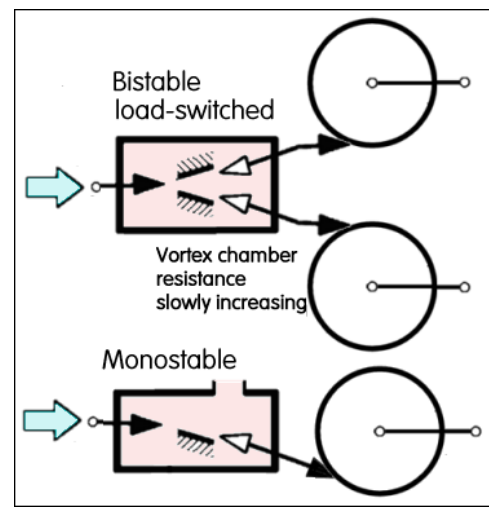

Figure 19 The basic principle of the recent relaxation oscillator $[13,14]$. The monostable version is similar to Fig. 13, accumulation delay by compressibility replaced by the spinning-up of rotation in vortex chamber.

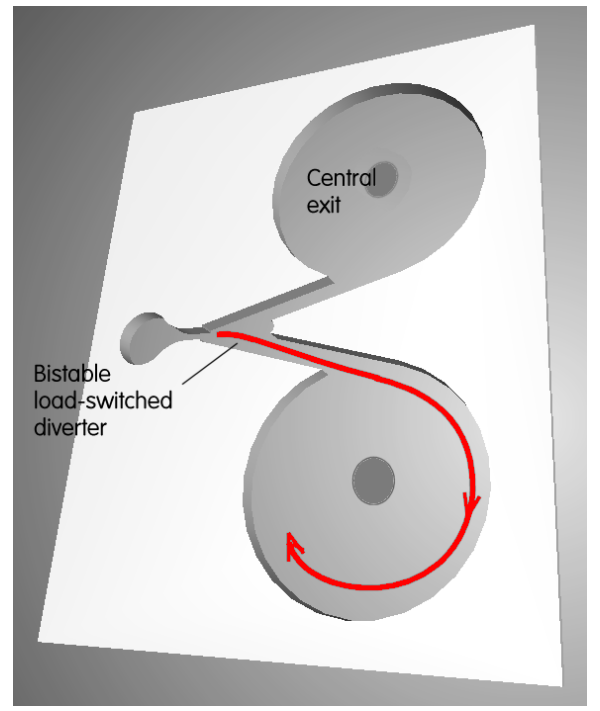

Figure 20 The main plate of fluidic oscillator according to $[14,13]$. In experimental evaluation it was capable of oscillating at extremely low frequencies. Symmetric configuration with bistable switching (top of Fig. 19).

The spin-up time delays in vortex chamber is a quite slow process and yet the oscillator is quite compact. Instead of the two parallel flowpaths of Fig. 15, with the division at the supply inlet into the circuit, there is here upstream from the vortex chambers the common jet-type diverter. It is load-switched when the rotation in the active vortex chamber attains the speed at which the Coanda effect cannot overcome the centrifugal force.
A demand for the low-frequency oscillators arose recently in bioreactors with supply of $\mathrm{CO}_{2}$ to cultivating algae in bioreactors [18] developed for the potential production of liquid fuels. The low frequency makes possible a return of generated microbubbles into the passage inside the aerator during each cycle. This prevents the microbubbles from their conjunction and keeps them small - with advantageous surface-area to volume ratio.

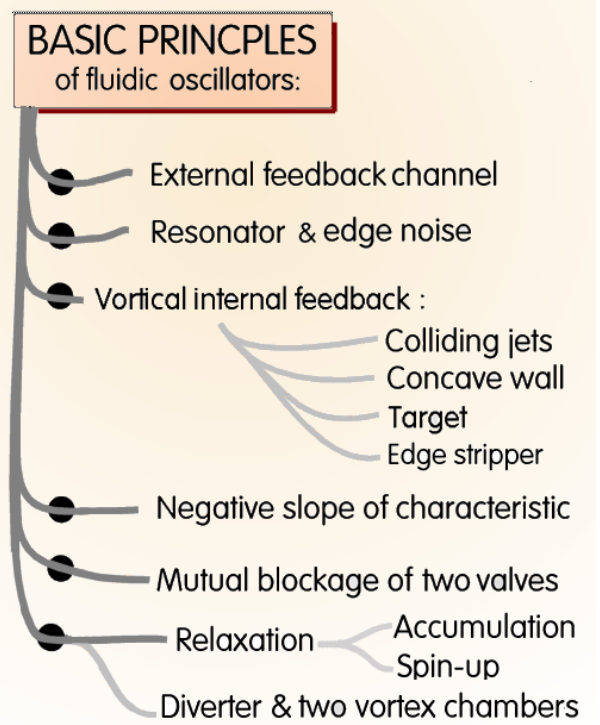

Figure 21 Taxonomic tree of oscillators arranged on the basis of operation mechanism principles.

\section{Classifications criteria for taxonomic trees}

As is certainly apparent already from the brief survey above that mere enumeration of the known oscillator principles produces a quite long list. It would be useful to have for general orientation in such lists a tree of mutual relations. As is the general problem in taxonomy, the key difficulty lies in the choice of the applied classification criterion. The resultant trees are different when formed on different bases.

Somewhat resembling the classification of organisms in biology, in a response to requests the present author has found three alternative approaches to the classification:

I) Feedback principle criterion

II) Criterion based on the working mode

III) Number of amplifiers

\subsection{Classification according to the used feedback} principle

The simple taxonomic tree resulting from the consideration of working principles discussed in the previous Sections of this paper is presented in the diagram Fig. 21.

No particular ordering of the items was there applied and this may result in certain shortcomings. For example, the final part of the previous Section 5 discussed an 
oscillator related to the relaxation type as well as to the oscillator with mutual blockage of two amplifiers. Such cross-linkage, however, was difficult to include the the tree graph - and the solution in Fig. 21 is mere addition of yet another function principle, related to the relaxation oscillators.

\subsection{Classification by the number of active devices (amplifiers)}

In another attempt at the classification as the basis for setting up the tree, presented in Fig. 22, the basic criterion applied was the number of active elements i.e. the number of fluidic amplifiers as devices that could be separately investigated. There is no such device (at least not immediately apparent) in the tree branches from S1 to S3 in Fig. 22. Also the load-switched diverter in the branch $\mathrm{R}$ would meet difficulties if it were to be used separately as an amplifier - despite the fact that some amplification effects are present.

\subsection{Working mode classification}

In another, earlier attempt at setting up a graphical survey of oscillators, the relations between the tree members were taken more into focus - as is apparent in its reproduction here in Fig. 23. More attention is there given to the relaxation switching, which seemed to offer a number of advantages for the algae growing bioreactors [18]. The permutations of bistability and monostability on one hand - and switching by a signal transported through the feedback channel vs. the load-switching has led to the undesirable crossing of the relevant branches.

The tree as presented in Fig. 23 was used as a basis for detailed discussions in which the coloured number indicating the classification was helpful. An example of such local detailed development of the tree is presented in the following Fig. 24. Individual configurations are there drawn schematically. As in the schematic representations above, the black (filled) triangles in this representation represent the nozzles (converting pressure energy into increased flow velocity) while the white (empty) triangles are represent diffusers (flow slowed down while pressure rises). Because of the flow direction changes of some of the represented flows, these representations have to be considered with care. Also schematically represented are the Coanda-effect attachment walls.

The devices shown in the top row of Fig. 24 oscillate being switched by flow from the perpendicularly oriented control nozzles. These nozzles are connected to the feedback loops (i.e. this genera in the tree are equal to the genus $A$ as shown in Fig. 3). In the middle horizontal row are relaxation type oscillators, with load-switched amplifiers and accumulation chambers $C$ in which air (or a compressible fluid in general) is accumulated for a part of the cycle to be released in the subsequent part.. The bottom row cases are the present author's novel configurations, with load-switched diverters and vortex amplifiers - in some cases reduced to mere passively behaving vortex chambers. As for the vertical columns, in the left-hand column are monostable relaxation oscillators, in the middle column are symmetrically switched configurations with bistable diverter and, finally, at right are cases of anti-parallel oscillators with mutually blocking two fluidic amplifiers. They are currently investigated for microbubble generation applications - which were made possible (and in some cases economically feasible) by oscillation generated by fluidics.

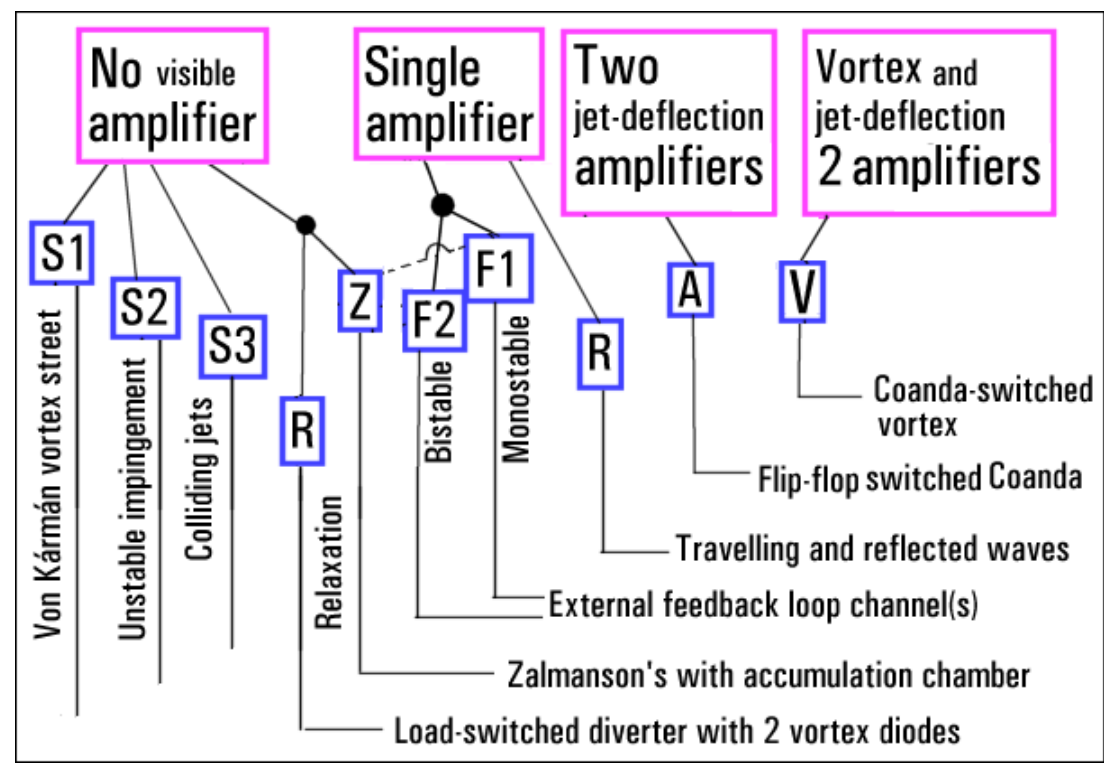

Figure 22 Taxonomic tree of fluidic oscillators with classification based on the number of active (i.e. amplifying) devices. 


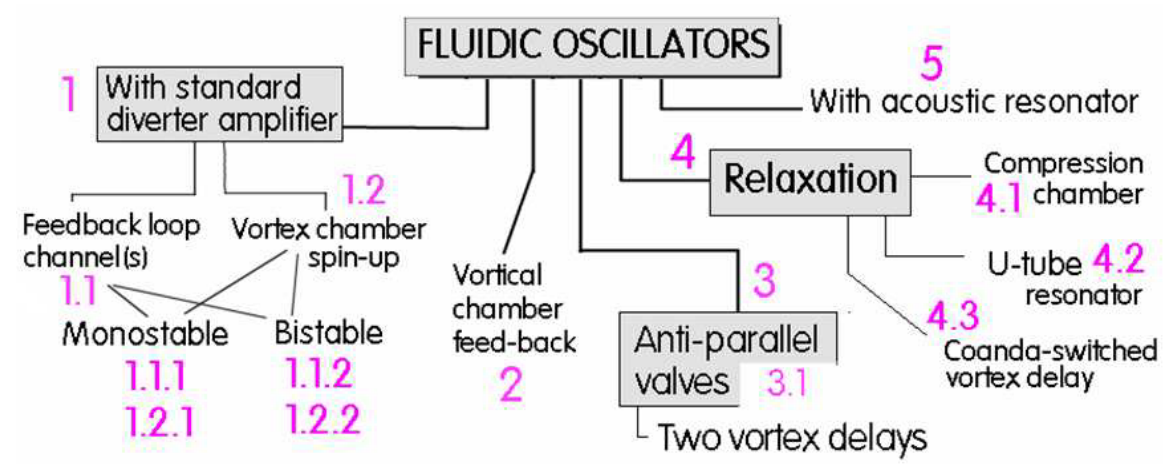

Figure 23 Alternative taxonomic tree of fluidic oscillators with classification numbering.

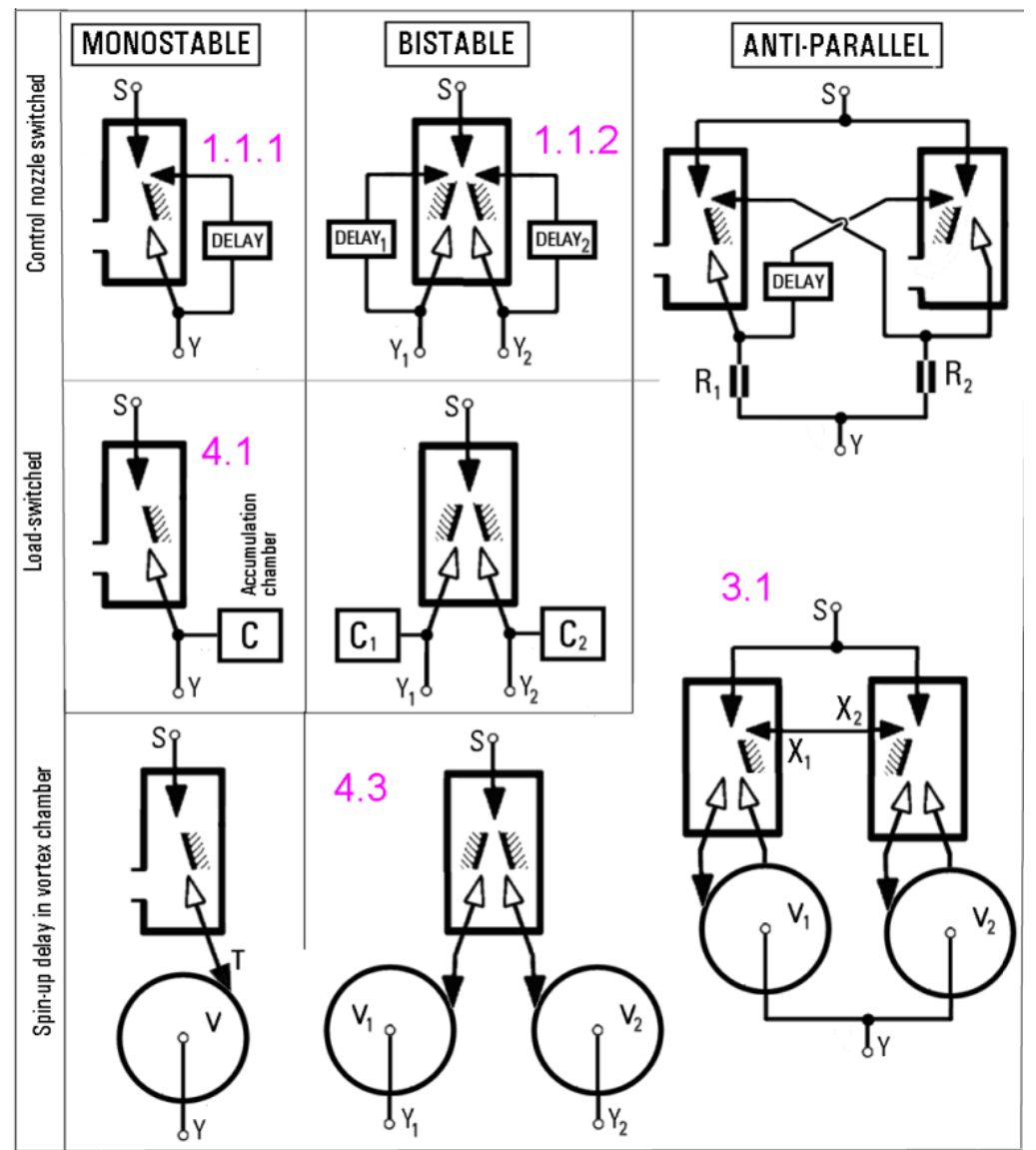

Figure 24 A part of taxonomic tree: table of genetically related oscillator configurations with single-jet diverter bistable and monostable amplifiers.

\section{Conclusions}

The starting point in scientific investigations is listing of investigated objects. The next one is to classify them and put them into order, using it in subsequent detailed studies. Fluidic oscillators are objects that recently became of importance. The number of applicable

\section{Acknowledgements}

Author obtained support from GAČR - Grant Agency of the Czech Republic, by research grant Nr. 13-23046S. There was also institutional support RVO:61388998. principles of flow pulsation is large and the mutual relations between them not clearly discernible. Following the analogous approach used in biological sciences, present author provided a list of working principles coming into consideration and set up an analogy to taxonomic trees.

\section{References}

1. V. Tesař, S. Zhong, F. Rasheed, AIAA Journ. 51397 (2013)

2. V. Strouhal. Annalen der Physik und Chemie 5 216251 (1878) 
3. C.-L. Sun, C.-Y. Sun C.-Y., Microsystem Technologies 17911 (2011)

4. O. Uzol, C. Camci, Proc. of 4th Internat. Symp. on Particle Image Velocimetry, paper 1029, Göttingen (2001)

5. Nakayama Y. et al., Bulletin of JSME 293313 (1986)

6. V. Tesař, Chemical Engineering Journal 155789 (2009)

7. H. Abraham, E. Bloch, Annales de Physique 9237 (1919)

8. R. W. Warren, US Patent 3,016,066 (1960)

9. L. A. Zalmanzon, US Patent 3,295,543 (1960)

10. C. E. Spyropoulos, Proc. of the Fluid Amplification Symp., Harry Diamond Laboratories, Washington D.C. (1964)

11. R. W. Warren, US Patent 3,016,066, 1960

12. V. Tesař, Czechoslovak Patent Nr. 262367 (1986)

13. V. Tesař, K. Peszynski, Smyk E., EPJ Web of Conferences 11402121 (2016)

14. V. Tesař, Czechoslovak Patent Nr. 306064 (2014)

15. V. Tesař, EPJ Web of Conferences 11402123 (2016)

16. R. Esaki, Y. Kurose Y., US Patent 3033714 (1957)

17. V.Tesař, C.-H. Hung, W. Zimmerman, Sensors and Actuators A 125159 (2006)

18. W.B. Zimmerman, B.N. Hewakandamby,

V. Tesař, H.C.H. Bandulasena, O.A. Omotowa International Sugar Journal 11290 (2010) 